\title{
Sensor Performance Assessment for Terra and Aqua MODIS Using Unscheduled Lunar Observations
}

\author{
Truman Wilson ${ }^{a}$, Amit Angal ${ }^{a}$, and Xiaoxiong Xiong ${ }^{b}$ \\ ${ }^{a}$ Science Systems and Applications, Inc., 10210 Greenbelt Road, Lanham, MD 20706, USA; \\ ${ }^{b}$ Sciences and Exploration Directorate, NASA/GSFC, Greenbelt, MD 20771, USA
}

\begin{abstract}
The Moderate Resolution Imaging Spectroradiometer (MODIS) has been in operation for over 18 and 16 years on the Terra and Aqua spacecrafts, respectively. In order to maintain long-term calibration stability over the life of each mission, MODIS uses a set of on-board calibrators as well as observations of the Moon and selected Earth-view targets. The lunar observations nominally occur in a narrow phase angle range, $55^{\circ}-56^{\circ}$, and use scheduled spacecraft maneuvers in order to bring the Moon into alignment with the MODIS space-view port. These observations are used to help characterize the MODIS scan-mirror response versus scan-angle. In addition to these scheduled lunar observations, MODIS also views the Moon through the space-view port without a spacecraft maneuver when the geometry is appropriately aligned. This occurs over a wider phase angle range, between $51^{\circ}-82^{\circ}$ degrees, than those of the scheduled moon observations. While the phase angle restriction of our scheduled observations provides consistency between the calibration events, the unscheduled Moon data can provide a valuable assessment of many calibration related investigations that use the Moon. In this paper, we compare the results of unscheduled versus scheduled lunar observations for several sensor calibration and performance assessments. These include the lunar calibration trending used to characterize the scan-mirror response versus scan angle and the electronic crosstalk correction of bands 27 - 30, which are currently used in the MODIS Level-1B data products, as well as sensor performance assessments such as band-to-band and detector-to-detector spatial registration.
\end{abstract}

Keywords: MODIS, lunar calibration, reflective solar bands, Moon, spatial characterization, Terra, Aqua

\section{INTRODUCTION}

The Moderate Resolution Imaging Spectroradiometer (MODIS) on board the Earth Observing System's (EOS) Terra (AM-1) and Aqua (PM-1) satellites is one of the key sensors used for long-term satellite-based climate studies. ${ }^{1}$ MODIS is a whisk-broom scanning radiometer with 36 separate spectral channels (bands) ranging in wavelength from $0.4-14.2 \mu \mathrm{m}$, which contain 20 reflective solar bands (RSB) and 16 thermal emissive bands (TEB). The bands are separated onto four focal plane assemblies (FPA) which group the bands by wavelength. These FPA groups are the visible (VIS), near-infrared (NIR), short- and mid-wave infrared (SMIR), and longwave infrared (LWIR). The layout of each of the bands on the FPAs can be seen in Figure 1. Each band consists of a linear array of detectors which are arranged in the instrument track direction (along the direction of satellite motion), with each of the bands placed on the FPA in the instrument scan direction (perpendicular to the track direction). Each MODIS scan spans $10 \mathrm{~km}$ in the track direction (nadir) and 2,330 km in the scan direction, at three different nadir resolutions, with bands 1 and 2 at $250 \mathrm{~m}$, bands $3-7$ at $500 \mathrm{~m}$, and bands $8-36$ at $1 \mathrm{~km}$. These bands contain 40, 20 and 10 detectors respectively, and sample at different rates in order to obtain pixels with equal resolution along-track and along-scan at nadir.

The MODIS instruments have been in operation since 1999 and 2002 on Terra and Aqua respectively. ${ }^{1}$ In order to maintain long-term consistency of the dataset over this time period, the MODIS detectors are calibrated on-orbit using a set of on-board calibrators (OBC) and selected external targets. The RSBs are calibrated using a solar diffuser (SD) with its corresponding solar diffuser stability monitor (SDSM), as well as using observations of the Moon and selected Earth-view targets to characterize the degradation of the scan mirror as a function of

Further author information: (Send correspondence to T.W.)

T.W.: E-mail: truman.wilson@ssaihq.com, Telephone: 13018672120 

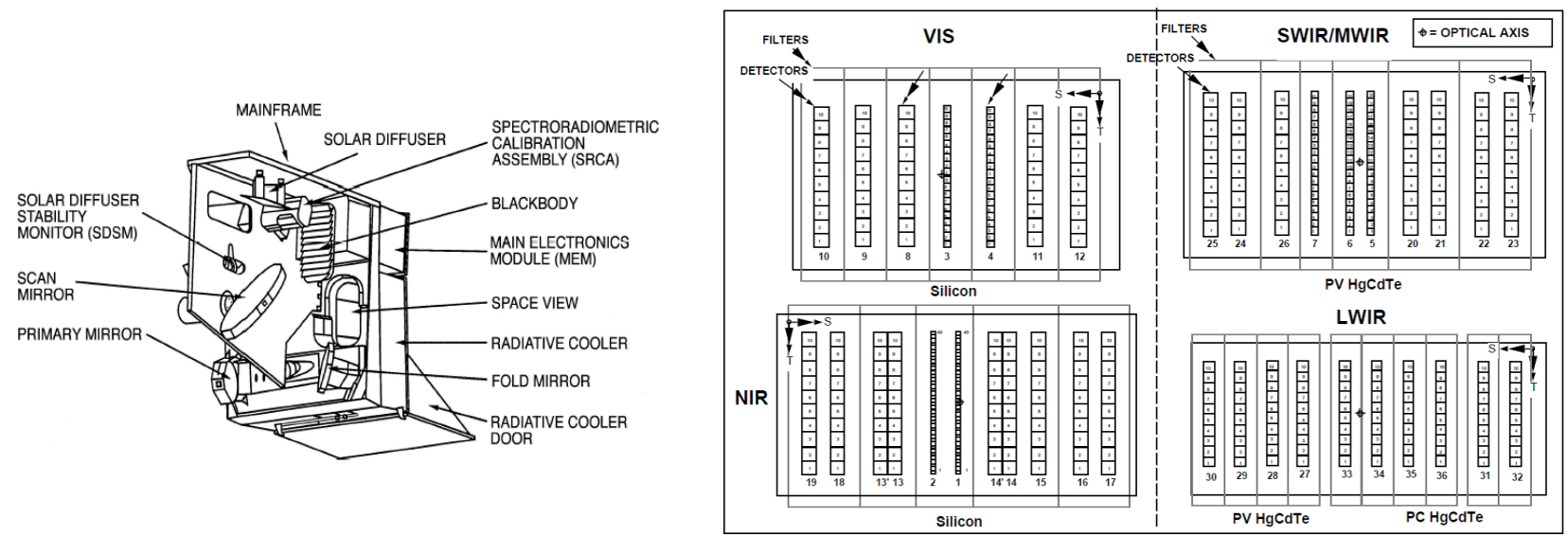

Figure 1. Diagram of the internal components of the MODIS instrument (left) including on-board calibrators and the space-view port, and the FPA layouts (right).

the angle-of-incidence (AOI) on the scan mirror. ${ }^{2}$ The TEBs are calibrated using a blackbody (BB) target. $^{3}$ For spatial and spectral characterization, both MODIS instruments have a spectroradiometric calibration assembly (SRCA) ${ }^{4,5}$ MODIS also has a space-view port, which is used for background subtraction and for viewing the Moon.

MODIS has a long history of using the Moon for various sensor performance assessments and calibration activities, such as the scan-mirror response versus scan angle (RVS) characterization, ${ }^{2}$ band-to-band (BBR) and detector-to-detector (DDR) spatial registration, ${ }^{6,7}$ and the characterization of electronic crosstalk between bands. ${ }^{8-11}$ The Moon is useful for these activities because it is a well-characterized radiometric target that is expected to have little change over the lifetimes of satellite missions and because it is a bright, well-resolved target against the dark background of space. The combination of these characteristics sets the Moon apart from both the OBCs and Earth-view targets, which also provide various forms of sensor performance assessments. ${ }^{12,13}$ For the calibration coefficients used in the MODIS Level-1B calibrated reflectance and radiance products, the lunar observations come from scheduled events where a spacecraft roll maneuver is performed in order to view the Moon in a narrow, consistent phase angle range from event-to-event. ${ }^{14}$ These observations occur on a near monthly basis with a nominal phase angle range of $55^{\circ}$ to $56^{\circ}$ and $-55^{\circ}$ to $-56^{\circ}$ for Terra and Aqua MODIS respectively (see Section 2).

MODIS can also observe the Moon when the position of the Moon happens to be in close alignment with the space-view port. ${ }^{15}$ This will only occur under the appropriate geometric configurations over a range of lunar phase angles for each instrument. These unscheduled observations provide an opportunity to do the same sensor performance assessments as the scheduled observations without the expense of planning and coordinating satellite maneuvers, which can affect the operation of the other instruments on-board the Terra and Aqua satellites. In this work, we will discuss the geometric requirements for unscheduled observations (Section 2) and we will analyze and compare the results of several on-orbit calibration activities using the scheduled and unscheduled Moon observations. In Section 3, we will analyze the trending gain of the sensor and discuss the consistency of the measured gain as it relates to the phase angle of the observation, which will give us insights into how model corrections of the lunar irradiance perform over a wide range of phase angles. In Section 4, we will analyze the spatial characterization results to see if unscheduled Moon observations produce similar results to that of the scheduled Moon, which has previously shown good consistency with the SRCA spatial characterization. ${ }^{7}$ Finally, in Section 5, we will compare the derived electronic crosstalk coefficients for bands $27-30$ to the values derived for MODIS Collection 6.1.9 


\section{GEOMETRY OF LUNAR OBSERVATIONS IN MODIS}

For both scheduled and unscheduled lunar observations, the Moon is viewed through the instrument space-view port (see Figure 1). For scheduled observations, an instrument roll maneuver is performed in order to bring the Moon into alignment with the space-view port at the desired time. ${ }^{14,16}$ For unscheduled observations, the Moon comes into alignment with the space-view port without a maneuver, and can occur for several consecutive orbits under the appropriate geometric configurations. ${ }^{15}$ For this work, we require that a full-disk image of the Moon is visible, which restricts the angular offset of the Moon with the space-view port to be within $\pm 1.2^{\circ}$ for the unscheduled observations. The MODIS space-view port is oriented $98.425^{\circ}$ away from nadir in the scan direction, which is $8.425^{\circ}$ away from the vector normal to the instrument orbital plane. Since this vector is close to the orbital plane normal, the region of space in which lunar observations can occur without an instrument maneuver is relatively narrow.

One of the most important parameters under consideration for this work is the lunar phase angle, which is defined as the angle between the Sun, Moon, and satellite. In this work, we will define negative phase angles as being during the waxing phase of the Moon (new Moon to full Moon) and positive phase angles to be during the waning phase (full Moon to new Moon). In Figure 2, we show the phase angle of the lunar observations versus the calendar day of the year for all of the years of each mission. For the scheduled lunar observations, most events occur in the range $55^{\circ}$ to $56^{\circ}$ and $-55^{\circ}$ to $-56^{\circ}$ for Terra and Aqua MODIS respectively. A few outlier events can be seen when the nominal observation phase angle would require a maneuver outside of the allowed roll angle range, which is up to $20^{\circ}$ away from nadir, so a different phase angle observation was selected. For the unscheduled lunar observations in which the full disk of the Moon can be observed, the phase angle range increases to $54^{\circ}$ to $82^{\circ}$ for Terra MODIS and $-52^{\circ}$ to $-80^{\circ}$ for Aqua MODIS. These observations can occur for many consecutive orbits with changing phase angles, which results in the vertical nature of the data shown in Figure 2 over phase angle on a given day.
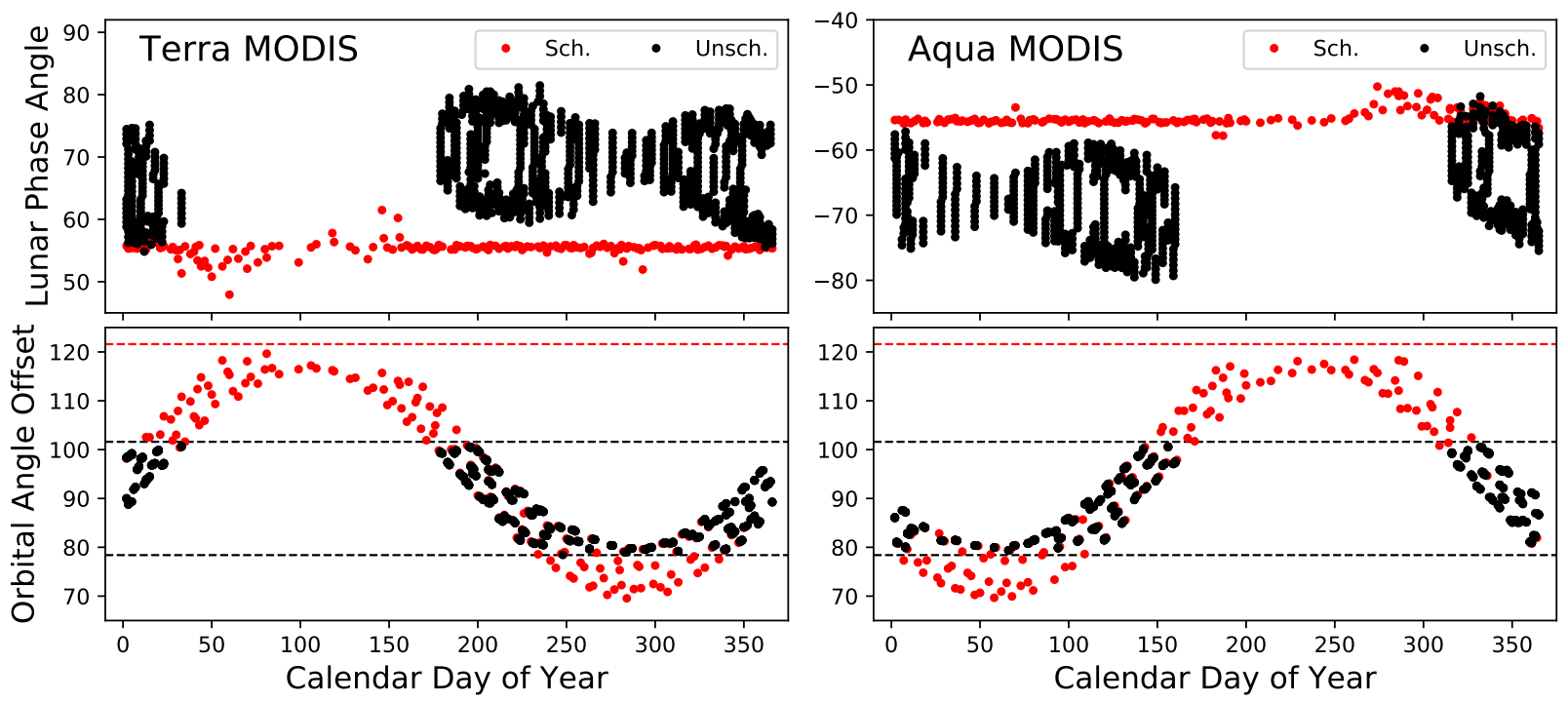

Figure 2. The top plots show the observed lunar phase angle as a function of the calendar day of year for Terra and Aqua MODIS. Scheduled and unscheduled observations are shown in red and black respectively. For each instrument, a "gap" in the data can be seen each year owing to repeated patterns in the orbital geometry of the instrument and Moon. The bottom plots show the angle between the orbital planes of the satellites and the Moon for the same events in the top plots. Unscheduled lunar observations can occur when the angle between the orbital planes is within $\pm 11.5^{\circ}$ of perpendicular. The dotted lines show the theoretical limits of the scheduled (red) and unscheduled (black) observations. For the scheduled observations, no lower limit is shown, as the observations do not approach this limit at the nominal phase angle. 
When examining the unscheduled observations for each instrument, we find that there is a period during each year where no unscheduled observations occur. This is because the satellite and lunar orbital geometries repeat annually, and the geometric requirements are not met during this time. In the lower plots in Figure 2, we plot the angular difference between the normal vectors that define the orbital planes of the satellites and Moon versus calendar date. Looking at this data, we find that unscheduled lunar observations occur only when the angle between these orbital vectors is within approximately $\pm 11.5^{\circ}$ of perpendicular, which is greater than the $8.425^{\circ}$ space-view port offset from the normal of the orbital plane with the $1.2^{\circ}$ extension to the field of view. This is because the satellite's orbit around the Earth, as opposed to having the instrument rotate at a fixed position at the center of the Earth, slightly increases the total range of the angular field of view of the space-view port as the satellite rotates throughout its orbit. ${ }^{14}$ We show the theoretical observation limits with dotted lines in Figure 2. For the scheduled observations, we have an additional $20^{\circ}$ degrees in which we can view the Moon because of the ability to roll the satellite.

To further illustrate the geometry of the unscheduled lunar observations, Figure 3 shows the relative position of the Earth, Moon, Sun and satellite for the lowest and highest phase angle observations for both Terra and Aqua MODIS. From Figure 3, we can see that the lunar observations occur for opposite phase angles of the Moon due to the opposite orbital trajectories of the two instruments (Terra is in a descending orbit, and Aqua is in an ascending orbit). Since each instrument is kept in a sun-synchronous orbit, and because the space-view port points near the normal of the orbital plane, the region of space where lunar observations can occur is relatively narrow. Therefore, there is no chance of a simultaneous observation of the Moon for both instruments and each instrument will only share a small portion of the illuminated lunar surface in its view (at a significantly different time and illumination angle). The observations for each instrument will span a range that is just under $30^{\circ}$ in phase angle.

The images in Figure 3 are for a single scan from band 1 and correspond to the observation times shown to the left of each image. These images show that for higher phase angle events, a lower fraction of the visible lunar surface is illuminated by the Sun. Also, the rotation seen in the lunar images between events is caused by the relative position and orientation of the satellite in its orbit during the time of the observation. For Terra

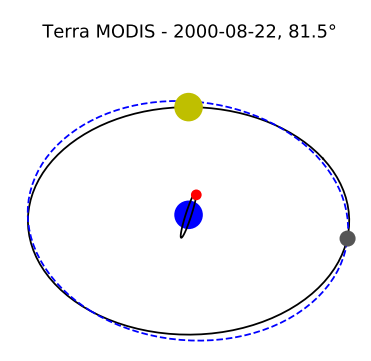

Terra MODIS - 2004-01-12, 54.8 ${ }^{\circ}$

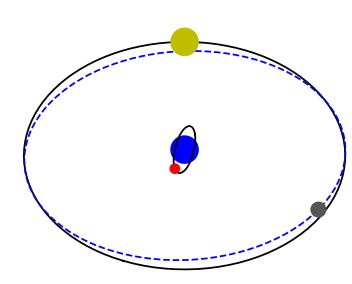

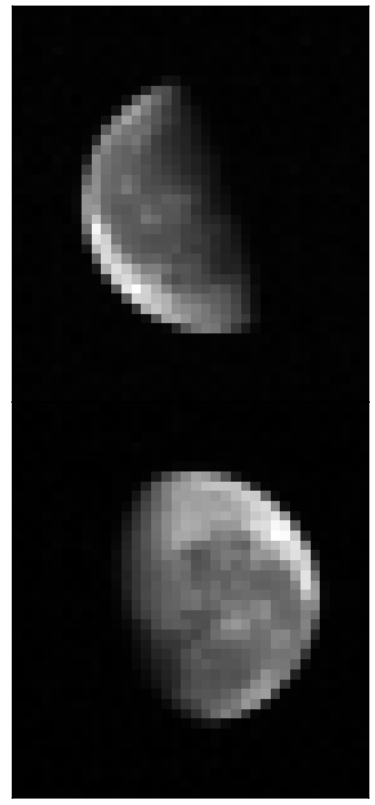

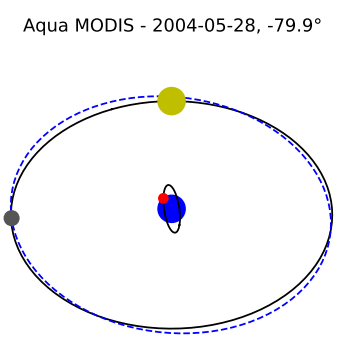

Aqua MODIS - 2009-11-28, $-51.8^{\circ}$
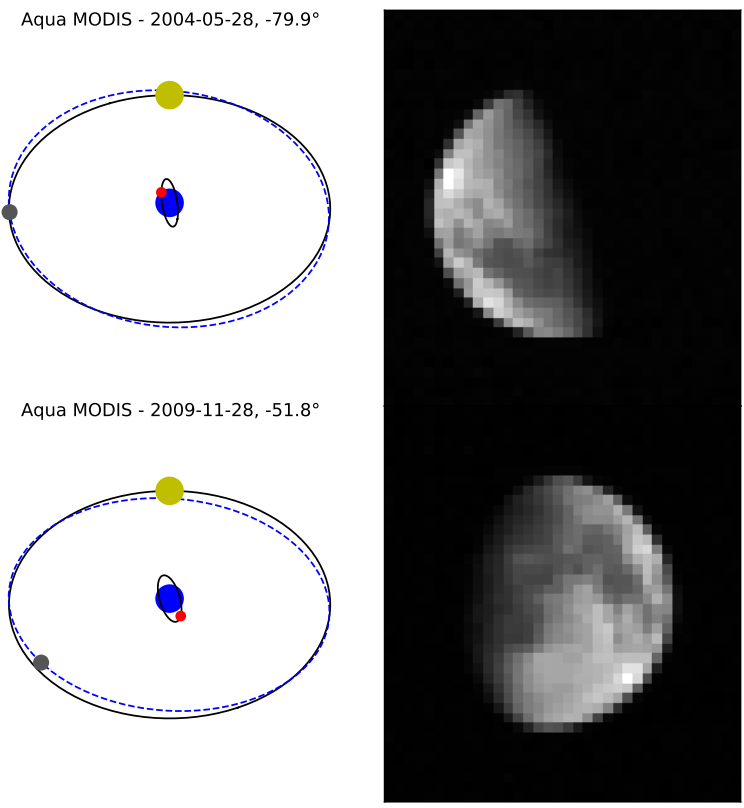

Figure 3. Orbital geometry and example images for the lowest and highest phase angle images for Terra and Aqua MODIS unscheduled lunar observations. For the orbital geometry, the Earth, Moon, Sun and satellite are shown as the blue, grey, yellow and red dots respectively. The lines show the respective paths of the Sun, Moon, and satellite relative to the Earth (not to scale). The corresponding images for band 1 (single scan) in MODIS are shown to the right of geometric plots. 
MODIS, the view of the Moon is mostly of the western hemisphere (in selenographic coordinates), while for Aqua MODIS, the view is of the eastern hemisphere.

The observation geometry will be particularly important for the calculation of the on-orbit gain trending using the Moon, as discussed in Section 3. In order to compare the data from the observations between events, we apply model corrections from Reference 17 which depend on the precise view geometry of the event. As we will show, for Terra and Aqua MODIS, the correction applied from the model will behave differently at different wavelengths for each instrument owing to the different observation geometries between the two instruments.

\section{LUNAR CALIBRATION}

For MODIS on-orbit operations, observations of the Moon are used to characterize the degradation of the scanmirror RVS. ${ }^{2}$ These observations occur through the space-view port, which is at $11.2^{\circ}$ AOI on the MODIS scan mirror compared to $50.25^{\circ}$ of the SD. In order to facilitate the use of these measurements for the MODIS RVS calculation, we calculate the trending gain of each band from the lunar observations using the pre-launch calibration coefficient values, $m_{1}^{p l}$, and the appropriate geometric correction associated with the geometry of the lunar observation. The details of the implementation of the lunar calibration in the on-orbit MODIS calibration algorithm can be found in Reference 14. For the purpose of the unscheduled lunar observation comparison, the discussion of the methodology will be limited to only the calculation of the sensor gain. Also, for this work, we will only consider MODIS scans where the full disk of the Moon can be observed for both the scheduled and unscheduled Moons, similar to lunar calibration methodology for S-NPP VIIRS. ${ }^{18}$ By doing this, we can remove pixel oversampling from consideration, which would be needed if integrating all lunar scans including partial images. ${ }^{14}$

The relative, band-averaged $(B)$ gain as a function of time $(t)$ can be written as:

$$
G(B, t)=\frac{1}{f_{n}(B, t) \cdot N_{S}(t)} \sum_{D, S, F, S F} m_{1}^{p l}(B, D, S F, M S(S)) \cdot d n^{\dagger}(B, D, S, F, S F, t)
$$

where $B, D, S, F$ and $S F$ are the band, detector, scan, frame and subframe of a given pixel respectively, $d n^{\dagger}$ is the background-subtracted digital counts of the observation after an instrument temperature correction has been applied, and $M S$ is the scan-mirror side, which alternates each scan. $N_{S}$ is the number of scans with a full lunar image for a given observation, the total number of which will vary based on the observation geometry. The term $f_{n}$ is the geometric normalization factor, which will allow us to make direct comparisons between lunar observations that have different observation geometries. It is comprised of geometric correction terms related to the Sun-Moon distance, Moon-satellite distance, lunar phase angle correction and lunar libration angle correction. The libration and phase angle corrections are computed using model data developed by Kieffer et. al. in Reference 17 .

For bands $13-16$, the overall gain is relatively high, which causes some pixels to be saturated when viewing the Moon. A simple integration of the pixel values would produce inconsistent results over observations at different phase angles, where the total number of saturated pixels is expected to change. Therefore, a correction is applied to the saturated pixels using the data from band 18 as a reference. ${ }^{19}$ Since the MODIS pixels through the space-view port are spatially co-registered, we apply the correction by evaluating the ratio of the unsaturated co-registered pixels and multiplying this ratio by the value of band 18 at the pixel location of the saturated pixels in bands $13-16$. Using these replacement pixels, we can achieve a more consistent comparison between the scheduled and unscheduled lunar observations in bands $13-16$. For bands $5-7$ and 26 , which are short-wave infrared (SWIR) bands, there is a well-known crosstalk contamination issue which has a significant effect on the lunar calibration results. ${ }^{20}$ For this work, we will ignore the SWIR bands and focus on the VIS/NIR bands.

The gain comparison results for Terra and Aqua MODIS can be seen in Figures 4 and 5, respectively. For each band, the gain is normalized to the value of the first scheduled lunar observation. In each figure, the bands are grouped by wavelength (see Figure 6). The results of the scheduled lunar observations are shown using solid lines, with the unscheduled results plotted over these lines as circles. With the geometric correction from Reference 17, we can see that for each band, the trending gain between unscheduled and scheduled lunar observations are in 

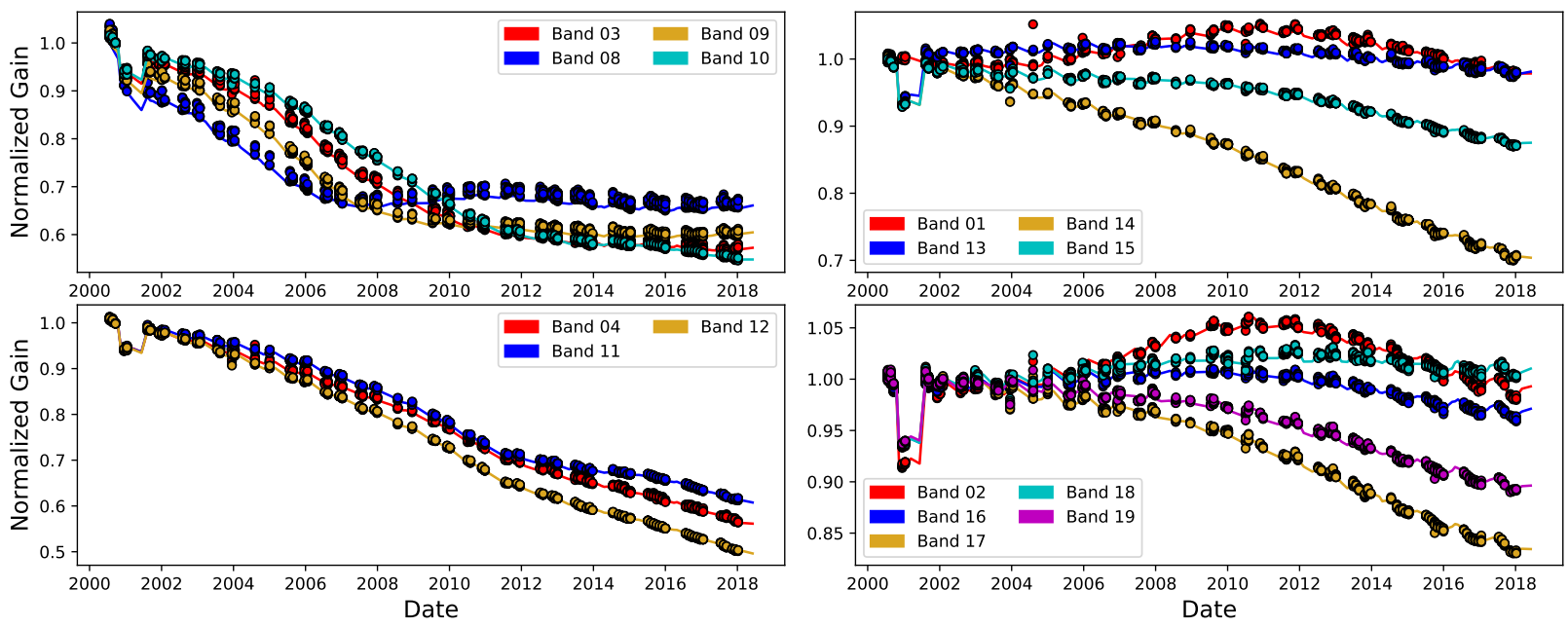

Figure 4. Gain comparison between scheduled (solid line) and unscheduled (circles) lunar observations for Terra MODIS. The bands in each plot are grouped by wavelength (see Figure 6). Each data point is normalized to the first scheduled lunar observation. The change in gain seen around 2001 is associated with a change in the detector electronics at this time.
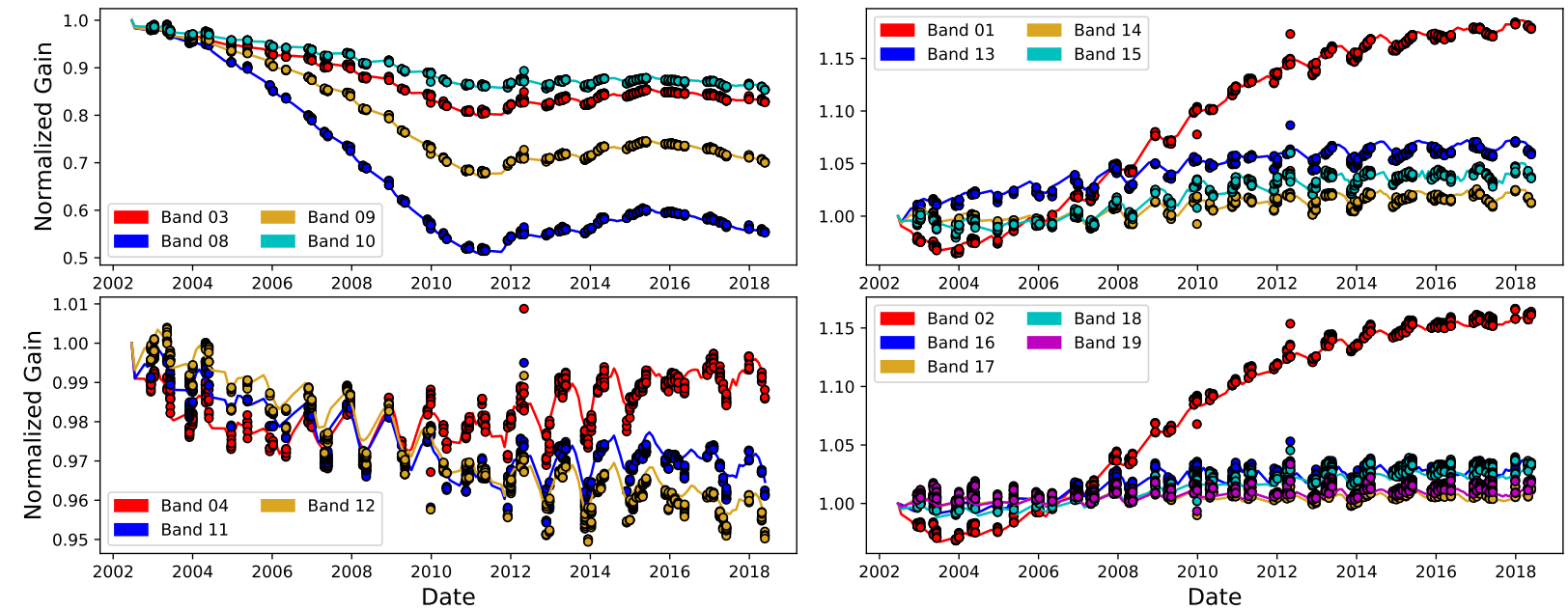

Figure 5. Gain comparison between scheduled (solid line) and unscheduled (circles) lunar observations for Aqua MODIS. The bands in each plot are grouped by wavelength (see Figure 6). Each data point is normalized to the first scheduled lunar observation.

good agreement over the mission lifetime. When comparing the gain between the same bands in Aqua and Terra over their respective missions, we note that for most bands the gain change for Aqua is smaller than that of Terra. This is reflected in the different scales used in Figures 4 and 5, particularly for bands 4, 11, and 12.

While the trending data matches well, for some bands the measured gain is slightly higher for the case of unscheduled lunar observations. To analyze this, we made a comparison of the relative gain ratio of unscheduled/scheduled lunar observations as a function of phase angle. First, we interpolate the gain of the scheduled results onto the times of the unscheduled results and take the ratio of the gain of the unscheduled observation with that of the interpolated value. We then group the data into bins representing a $1^{\circ}$ width in lunar phase angle and average the results. In Figure 6, we plot the ratio for each band for four selected phase-angle bins as a function of the band's center wavelength. 

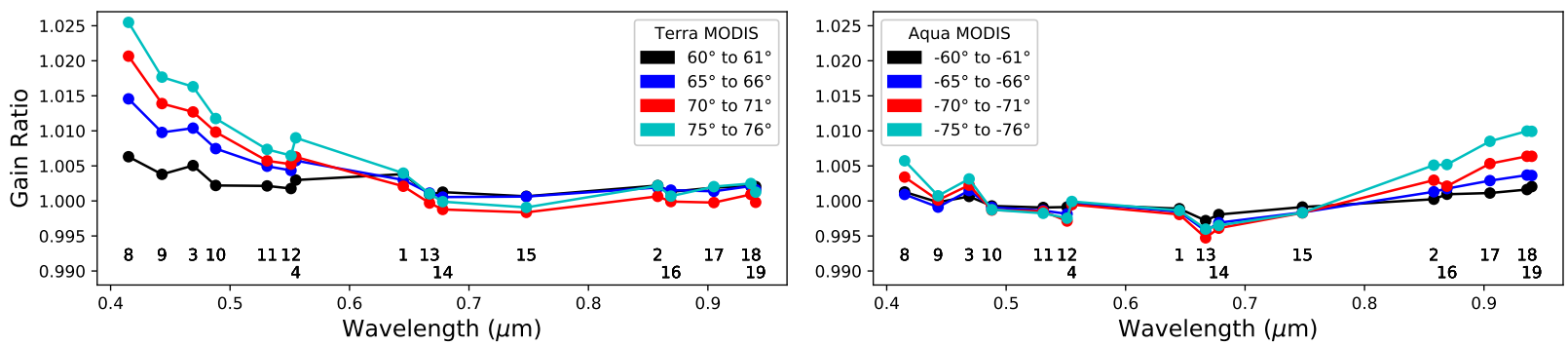

Figure 6. Relative gain ratio for unscheduled/scheduled lunar observations as a function of wavelength for Terra (left) and Aqua (right) MODIS. The observations were binned and averaged in 1 degree increments over the phase angle. Four selected phase angles are are shown for each instrument, with ratios relative to the scheduled observations, which occur between $\left[55^{\circ}, 56^{\circ}\right]$ and $\left[-55^{\circ},-56^{\circ}\right]$ for Terra and Aqua MODIS respectively. At the bottom of each plot, the band number is listed at its corresponding wavelength.

For Terra MODIS, there is a clear increase in the measured gain for bands at low wavelength as the phase angle increases, with changes greater than $2.5 \%$ for band $8(412 \mathrm{~nm})$ at phase angles greater than $75^{\circ}$. At higher wavelengths, the results are more consistent over the broad range of lunar phase angles that we observe for the unscheduled Moon data. This consistency might indicate that the model parameters capture the changes in the expected lunar reflectances at different phase angles better at higher wavelengths for the part of the Moon that is observed by Terra MODIS. However, this should be investigated more closely to remove other potential instrument effects. For Aqua MODIS, the overall change is smaller than what is seen for Terra MODIS. However, there does appear to be a larger change in the measured gain at higher (magnitude) phase angles for the higher wavelength bands, up to around 1\% for bands 17-19 (>900 nm). The lower wavelength bands of Aqua MODIS also appear to have a change in the gain ratio with phase angle, but this change is approximately a factor of 5 lower than what is seen for Terra MODIS.

By comparing the measured gains between the scheduled and unscheduled lunar observations, we have shown that the unscheduled observations capture the trend that is measured in the scheduled observations well. However, for some wavelengths, a narrow restriction in the phase angle range for comparison between observations would be best to maintain the consistency of the trending results.

\section{BAND-TO-BAND/DETECTOR-TO-DETECTOR REGISTRATION}

Since the Moon is well-defined, bright target against the dark background of space, it can be used effectively to do spatial characterization of satellite remote sensing instruments. ${ }^{6,7,21}$ The MODIS bands are spatially separated on the FPAs, and therefore, characterization of the spatial co-registration of the bands is of vital importance since the Earth-view images require shifts between the images of different bands in order to make pixel-by-pixel comparisons. Each MODIS instrument is equipped with an on-board SRCA which can be used for spectral, spatial, and radiometric calibration. ${ }^{4,5}$ In previous work, a comparison of spatial characterization using scheduled lunar observations and the SRCA showed good agreement between the two methods. ${ }^{7}$ For this work, we will focus on a comparison of the results between scheduled and unscheduled lunar observations, with the results updated through the beginning of 2018.

For MODIS observations of the Moon, the geometry is such that the Moon moves slowly across the FPA from scan to scan, which allows us to create images of the Moon versus scan and frame for each individual detector, as seen in Figure 7. From each of these images, the horizontal and vertical profiles for each detector can be calculated by integrating over the other dimension. The centroid position, $C$, in the scan $(S c)$ and track $(\operatorname{Tr})$ direction can then be calculated for each of the profiles using the following equations:

$$
C_{S c}(B, D)=\frac{\sum_{F}\left(\sum_{S} d n(B, D, S, F)\right) F}{\sum_{S, F} d n(B, D, S, F)}
$$



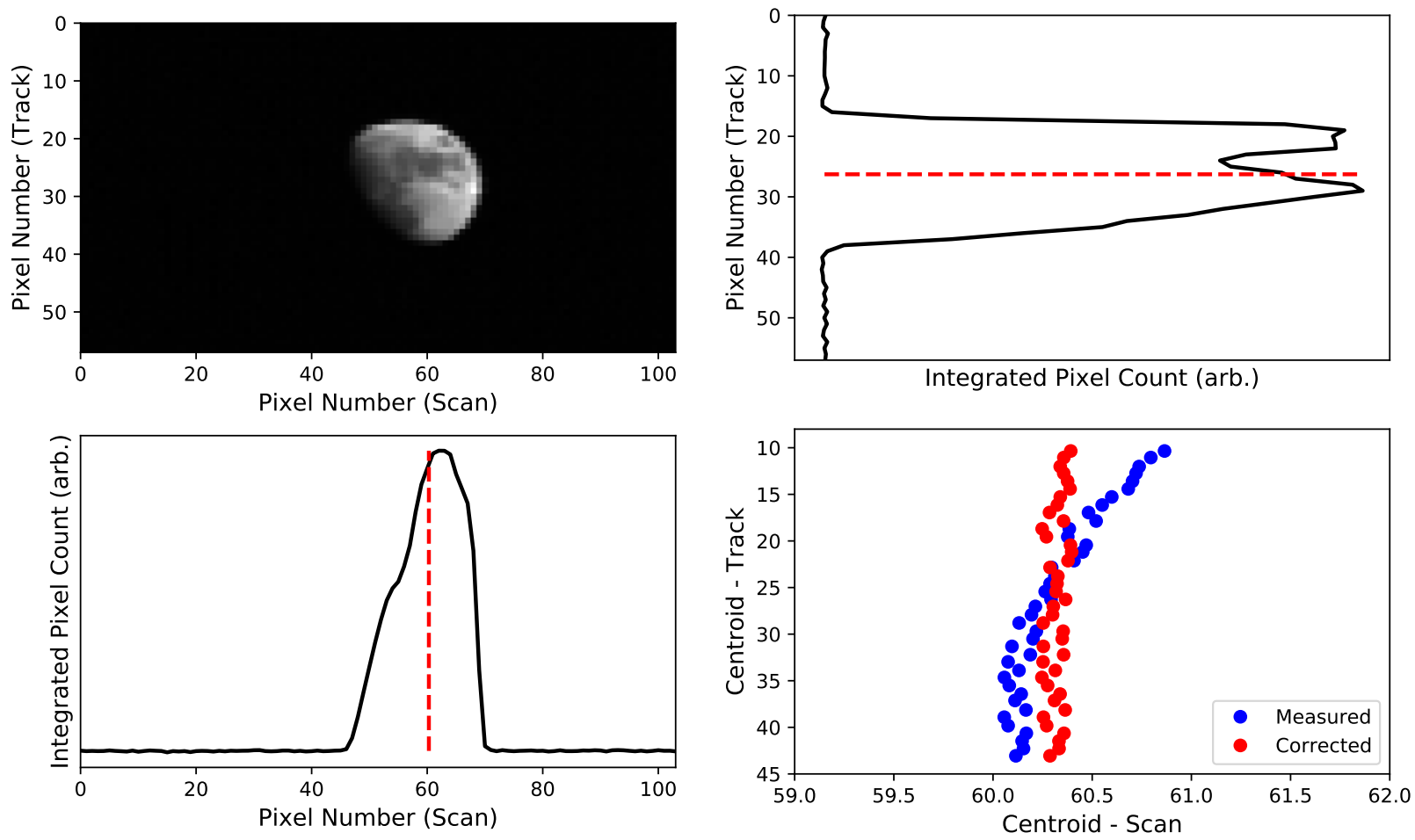

Figure 7. Example centroid calculation for a single detector image from Aqua MODIS band 1 from the scheduled lunar observation on February 6, 2017. The top left plot shows an image of the Moon from detector 20, with the scan and track directions oriented horizontally and vertically respectively. The top right plot shows the integrated signal over the scan direction as a function of track pixel. The bottom left plot shows the integrated signal over the track direction as a function of scan pixel. The centroid positions are marked with the dashed red lines. The bottom right plot shows the measured centroid positions for each detector in band 1 before (blue) and after (red) the lunar path and instrument attitude corrections.

$$
C_{T r}(B, D)=\frac{\sum_{S}\left(\sum_{F} d n(B, D, S, F)\right) S}{\sum_{S, F} d n(B, D, S, F)}
$$

where $d n$ is the background-subtracted digital counts and $B, D, S$, and $F$ are the band, detector, scan number, and frame number respectively. For this analysis, the subframes are not treated separately and are all used in a single image for each detector. An example of the centroid calculation can be seen in Figure 7. The integrated profiles and calculated centroids for the horizontal and vertical profiles of the image can be seen to the bottom and right of the image in Figure 7 respectively.

In the lower right plot of Figure 7, the calculated centroid position for each detector in band 1 is shown. For each detector, the centroid position changes in both the scan and track directions based on the relative motion of the Moon with respect to the orbital motion of the spacecraft during the observation. ${ }^{21}$ In the track direction, the centroid is expected to change at a rate that is the inverse of the oversampling factor. ${ }^{6,7,14}$ In the scan direction, the centroid position changes at a different rate for each detector and will have to be accounted for more carefully as seen below. Also, for scheduled lunar observations, the roll maneuver introduces an instrument attitude error in the scan direction as the spacecraft settles to its nominal pointing position.

To correct the centroid position in the scan direction, we calculate the relative position of the Moon in the spacecraft coordinate system using the NASA JPL Development Ephemerides, DE421, and SPICE. ${ }^{22,23}$ For a given observation time (different for each detector), the Moon's offset in the scan direction is calculated and 
then subtracted from the measured centroid position. This offset is derived from the angle of the lunar position vector in the scan direction relative to the space-view port, $\theta_{m}$. For scheduled lunar observations, we can also subtract off the expected pointing error, $\theta_{p}$, taken from instrument attitude data during the roll maneuver. The correction to the centroid position, $\Delta C_{s c}$, is given by:

$$
\Delta C_{s c}(B, D)=\frac{705 \mathrm{~km}}{R(B)} \tan \left(\theta_{m}(D)+\theta_{p}(D)\right)
$$

where $R(B)$ is the resolution of band $B$ in kilometers and $705 \mathrm{~km}$ is the nominal altitude of the satellite. These terms convert the angular offsets into pixel offsets which can be applied to the centroid positions. An example of this correction is shown along with the uncorrected data in Figure 7. Currently, the lunar path and instrument attitude correction is only applied to correct the centroid position in the scan direction. Further analysis of this correction will be the subject of future work.

From the corrected centroid data, the first spatial characterization we perform is for the BBR. We will note that since bands $13-16$ have saturation issues which rely on spatial co-registration for the correction, these bands will not be considered in this work. For these calculations, we use band 1 as the reference band, which by definition will have a BBR shift of $0 \mathrm{~km}$. To calculate the BBR, we take the average value of all of the centroid positions within each band and subtract them from the band 1 values. We then average all of the values for each year for the both the scheduled and unscheduled lunar observations. The along-scan and along-track BBR for both Terra and Aqua MODIS can be seen in Figures 8 - 11 .

The results of the BBR measurements show that there is good agreement between the scheduled and unscheduled lunar observations. Also, it shows that there is little change in the BBR of the MODIS sensors over their mission lifetimes. These results also show the same relative shifts between each of the bands when compared to previous results derived from the Moon data early in each mission. ${ }^{7}$ For the Aqua BBR along-track results, relatively larger values are seen in the BBR shift for many of the bands relative to band 1 as compared to the Terra results. These results are consistent with our expectations, as along-track shifts were introduced to the Aqua MODIS bands during the pre-launch testing phase. ${ }^{24}$

The second spatial characterization that we performed was for the DDR within each band. In this case, for each detector within a band, the centroid position is referenced to detector 1 within the same band. The same comparisons can be made in this case, but now we do not perform any band averaging. The results for both Terra and Aqua MODIS along-scan and along-track DDR can be seen in Figures 12 - 15 .

The results in these plots are projected in three dimensions, with the detector number and year as the horizontal axes and the DDR shift (in $\mathrm{km}$ ) as the vertical axis. As in the case of the BBR results, the data is averaged annually for both the scheduled and unscheduled lunar observations for comparison. For the measured

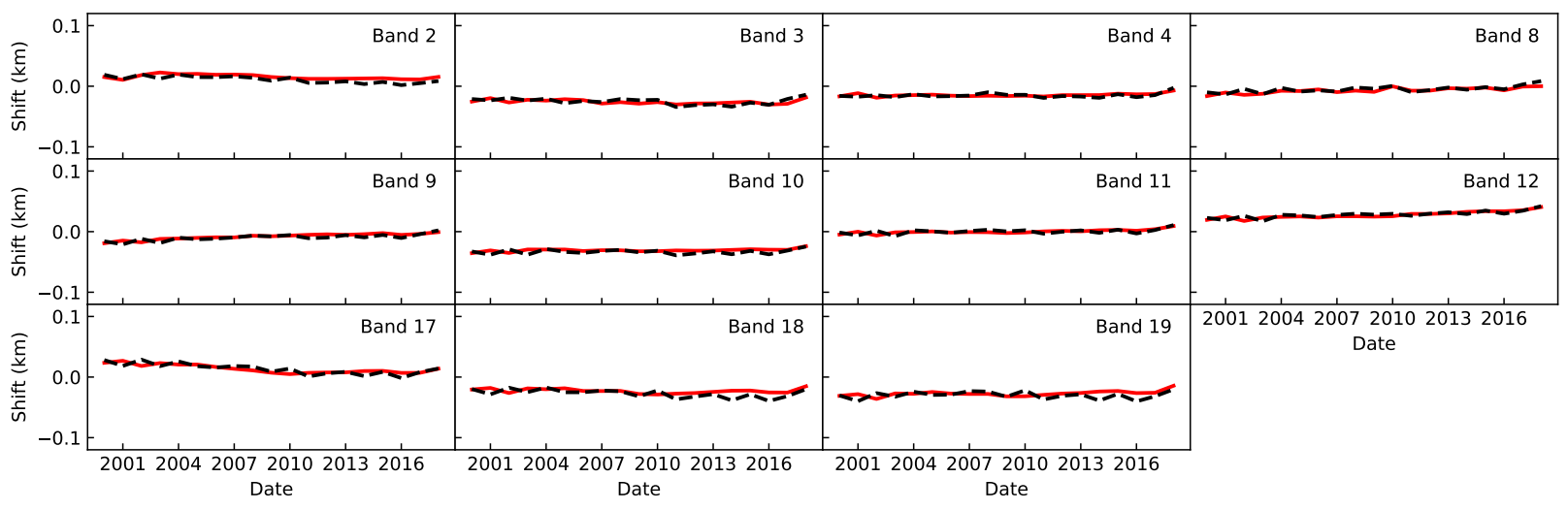

Figure 8. Terra MODIS BBR along-scan for scheduled (red, solid) and unscheduled (black, dashed) lunar observations. The data is the yearly average of all of the observations in each case. 
DDR along-scan, there is little change from year-to-year. Also, after the Moon path and spacecraft attitude correction is applied, the observed shift across each of the bands for both Terra and Aqua MODIS is small (typically less than $0.02 \mathrm{~km}$ ). This shows that these corrections do a good job of removing the detector differences in the centroid position in the scan direction across the entire mission for each detector. For band 2 in Terra MODIS, the results show a sharp decrease at detectors 8, 19 and 30, with the most significant decrease occurring for detector 19. For this band, there is a previously reported electronic crosstalk issue that can be observed in the lunar data which would affect the results of the DDR calculation. ${ }^{25}$ Band 1 in Terra MODIS also shows some signs of similar issues in the along-scan DDR for the same detectors, although at a much lower magnitude. These effects will need to be explored in the future.

The measured DDR along-track shows more change in general when compared to the along-scan results. Additionally, when comparing the scheduled to unscheduled results, we see a difference for the higher number detectors up to approximately $0.05 \mathrm{~km}$ for most bands. As stated before, the instrument path and attitude correction was only applied to the centroid positions in the scan direction. For the track direction, the pixel oversampling can be calculated and applied from the event geometry, however, the results seen here may indicate that an additional correction is needed to rectify the differences seen between the scheduled and unscheduled lunar results. The oversampling factor used for this work is fixed based on the geometry at the center time of the observation. Given the orbital dynamics of the observation, it may be necessary to use a time-dependent oversampling factor, and apply a path correction in the track direction similar to what was done in the scan direction. Also, for the case of the scheduled Moon, there is a small instrument attitude error in the track direction during the maneuver that may contribute to the differences we see here. The Aqua MODIS DDR along-track results also show some spikes in band 2 for detectors 8, 19, and 29, which may point to having a similar issue as that of Terra MODIS. We note that in this case though, the artifacts are only present in the along-track data, unlike Terra, where they can be seen in both the along-scan and along-track data. These effects will have to be investigated in future work.

Overall, the results of the spatial characterization using the unscheduled lunar data are in good agreement with the scheduled lunar data, with the exception of along-track DDR results. The path and attitude corrections applied in the scan direction greatly reduce the detector differences in the centroid position. A similar method for applying corrections to the track direction centroid positions will need to be explored.

\section{ELECTRONIC CROSSTALK CORRECTION}

Lunar observations have been used extensively in previous work in order to characterize the effects of electronic crosstalk on the MODIS sensors. ${ }^{8-11}$ In 2017, an electronic crosstalk correction was applied to bands $27-30$ to mitigate the effects of increasing crosstalk contamination throughout the mission in Collection 6.1. ${ }^{9}$ This correction greatly improved the MODIS imagery through a removal of detector-to-detector striping and a correction of

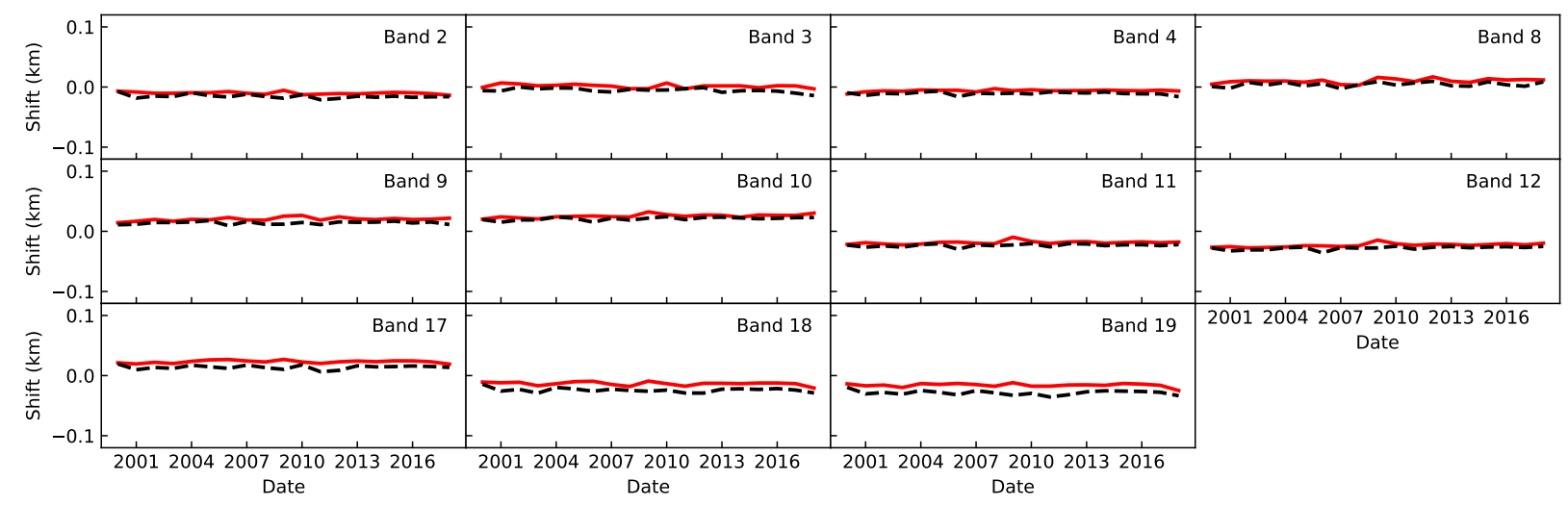

Figure 9. Terra MODIS BBR along-track for scheduled (red, solid) and unscheduled (black, dashed) lunar observations. The data is the yearly average of all of the observations in each case. 


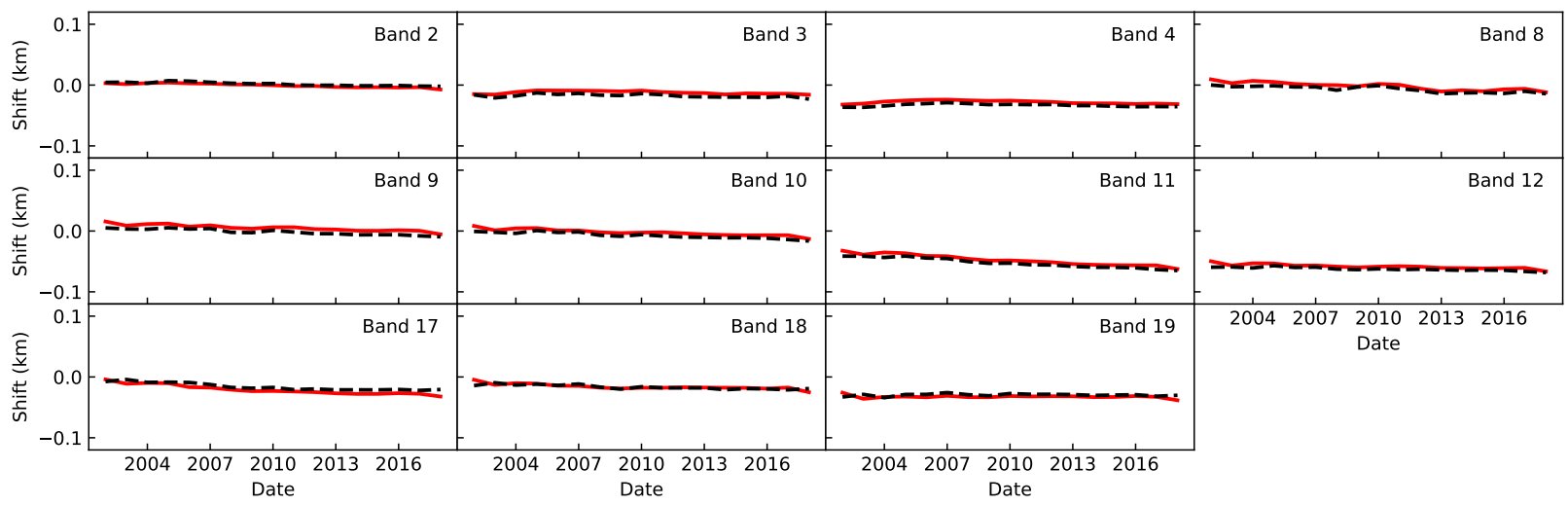

Figure 10. Aqua MODIS BBR along-scan for scheduled (red, solid) and unscheduled (black, dashed) lunar observations. The data is the yearly average of all of the observations in each case.

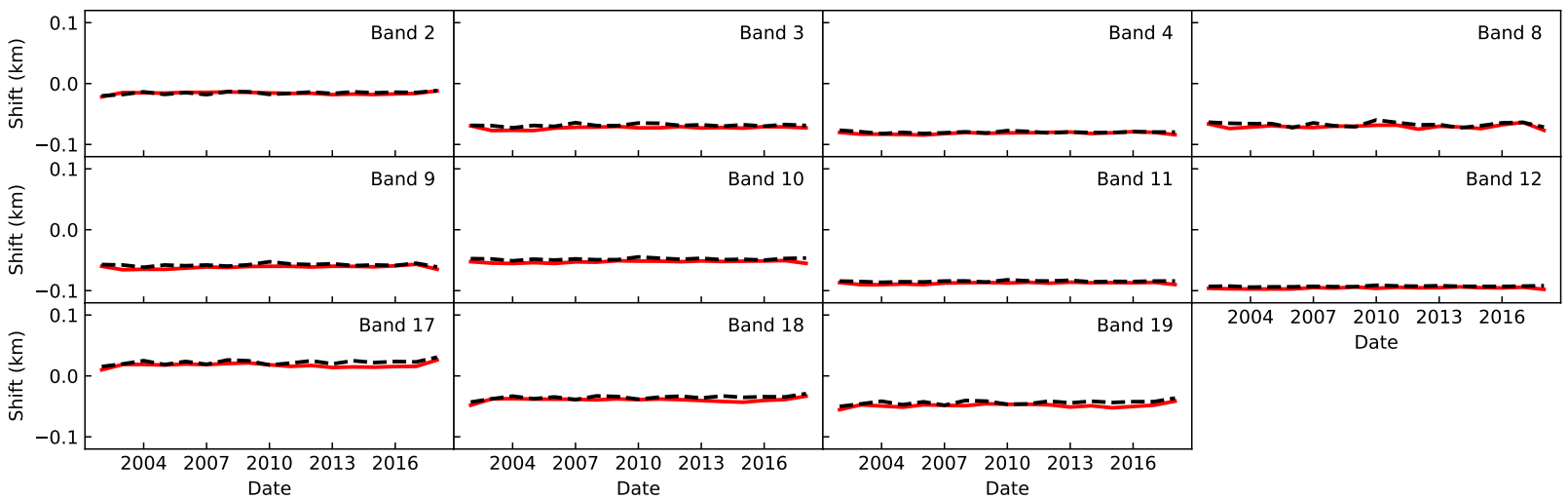

Figure 11. Aqua MODIS BBR along-track for scheduled (red, solid) and unscheduled (black, dashed) lunar observations. The data is the yearly average of all of the observations in each case.

the radiometric bias induced by the contamination. The corrected Level-1B data has been used to reprocess the downstream Level-2 and higher science products for Collection 6.1. The initial analysis shows an improvement to the science products as reported in Reference 26 .

The crosstalk contamination for a given "receiving" detector $(i)$ is modeled as being linearly-dependent on the measured signal from a given "sending" detector $(j)$. Using lunar data, we can derive a set of crosstalk coefficients, $c_{i, j}$, by evaluating the signal level of the space-view scene around the Moon compared to the expected background level. Given the spatial offset of the bands on the FPA, as seen in Figure 1, the contamination will appear at locations in the image that correspond to the spatial offsets between the sending and receiving bands. By comparing the value of the sending signal with the contamination level, the values of $c_{i, j}$ can be derived. For more details on the algorithm implementation, see Reference 9 .

With the crosstalk coefficients, the corrected signal for the $i$ th detector, $d n_{i}$, is given by:

$$
d n_{i}(S, F)=d n_{i}^{*}(S, F)-\sum_{j} c_{i, j} \cdot d n_{j}^{*}\left(S, F+\Delta F_{j}\right)
$$

where $d n^{*}$ is the measured (contaminated) signal level after background subtraction, $S$ and $F$ are the scan and frame number of a given pixel respectively, and $\Delta F_{j}$ is the frame offset between the sending and receiving bands corresponding to detectors $i$ and $j$. For MODIS data, this correction is applied at the pixel level for both 

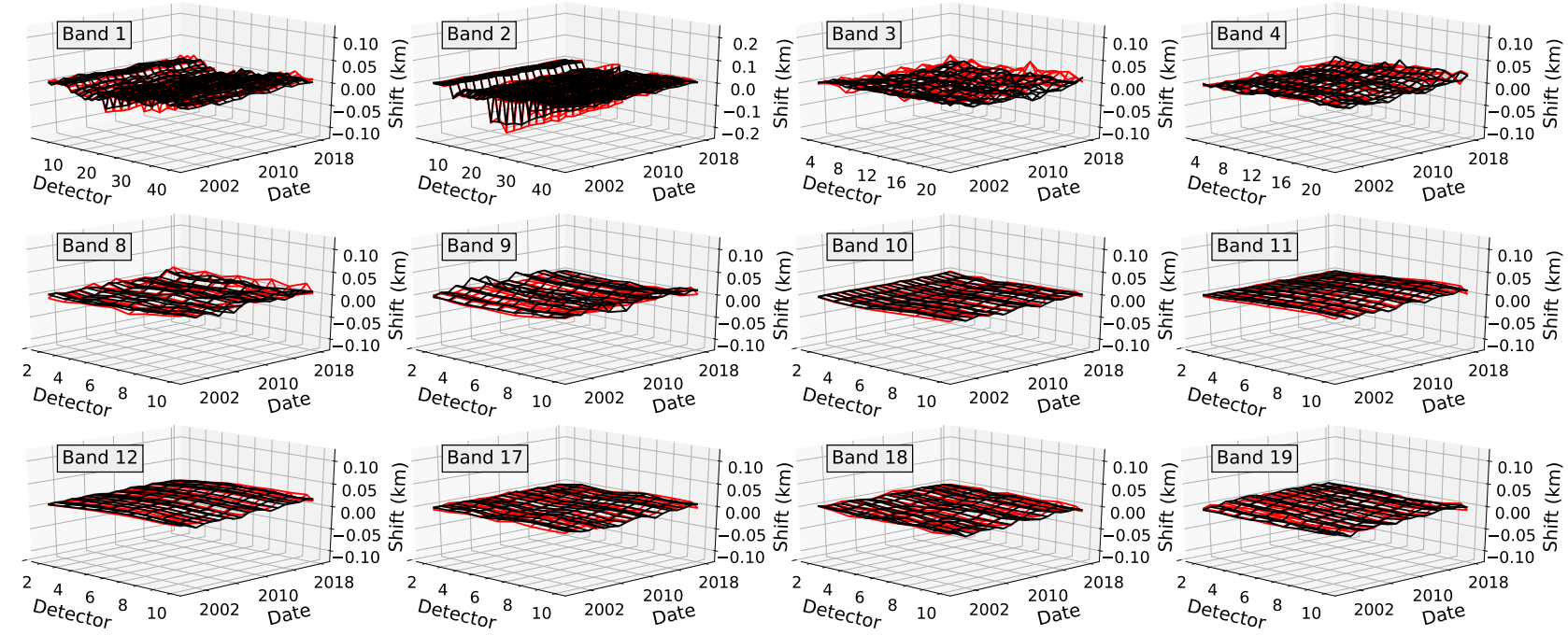

Figure 12. Terra MODIS DDR along-scan for scheduled (red) and unscheduled (black) lunar observations. The data is the yearly average of all of the observations in each case. Detector 1 is not shown because it is the reference for each of the other detectors.
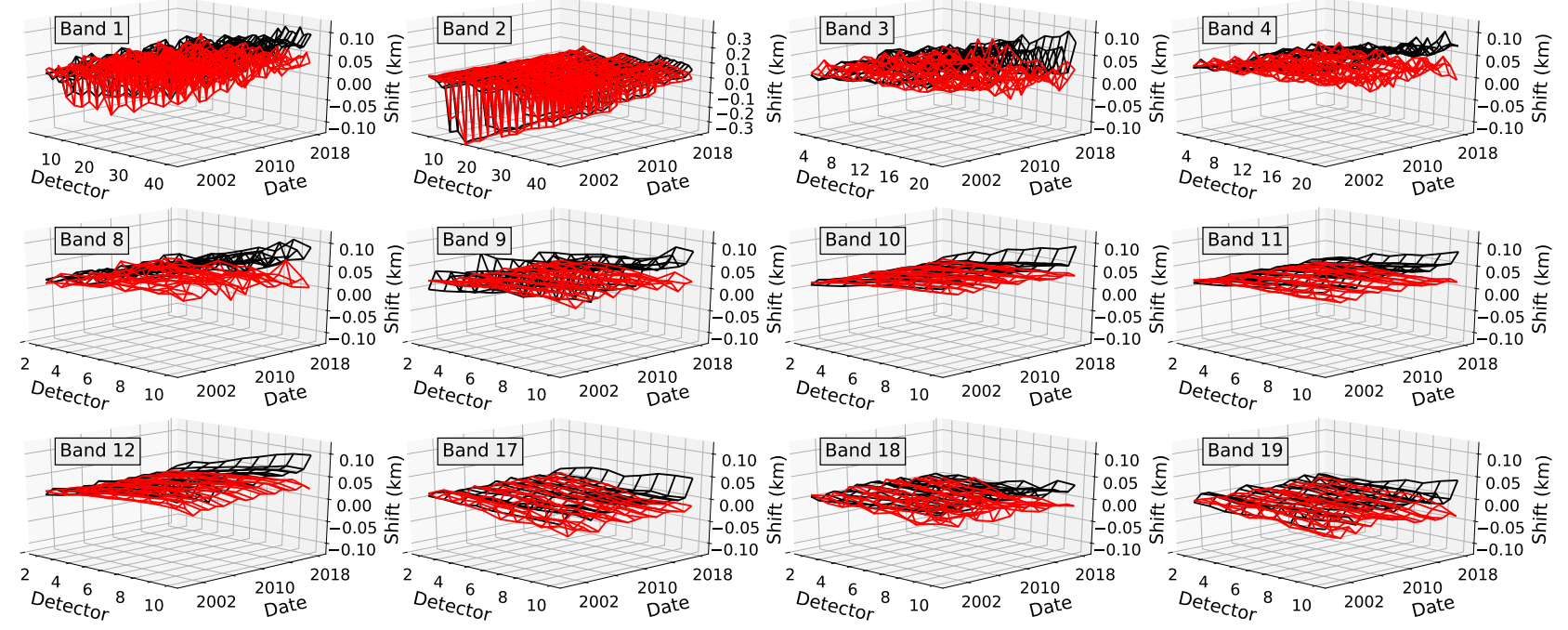

Figure 13. Terra MODIS DDR along-track for scheduled (red) and unscheduled (black) lunar observations. The data is the yearly average of all of the observations in each case. Detector 1 is not shown because it is the reference for each of the other detectors.

Earth-view and OBC data to create crosstalk-corrected Level-1B data. ${ }^{9}$ This crosstalk corrected data is then used to derive the higher level science data products in Collection $6.1 .^{26}$

The crosstalk correction coefficients for Collection 6.1 were derived from the scheduled lunar observations in order to leverage the existing calibration activities and to have regular spacing in the time-series of the coefficient values. While the unscheduled lunar observations have an irregular time-series as a result of the geometric restrictions for the observations, they can provide a check of coefficient values derived from the scheduled lunar observations in two key ways. First, since the unscheduled moons occur over many consecutive orbits, these observations can provide a check of the short-term time-stability of the coefficient values. Second, since there is saturation in the lunar signal for bands $27-30,{ }^{9}$ observations at a higher phase angle where the signal is less 

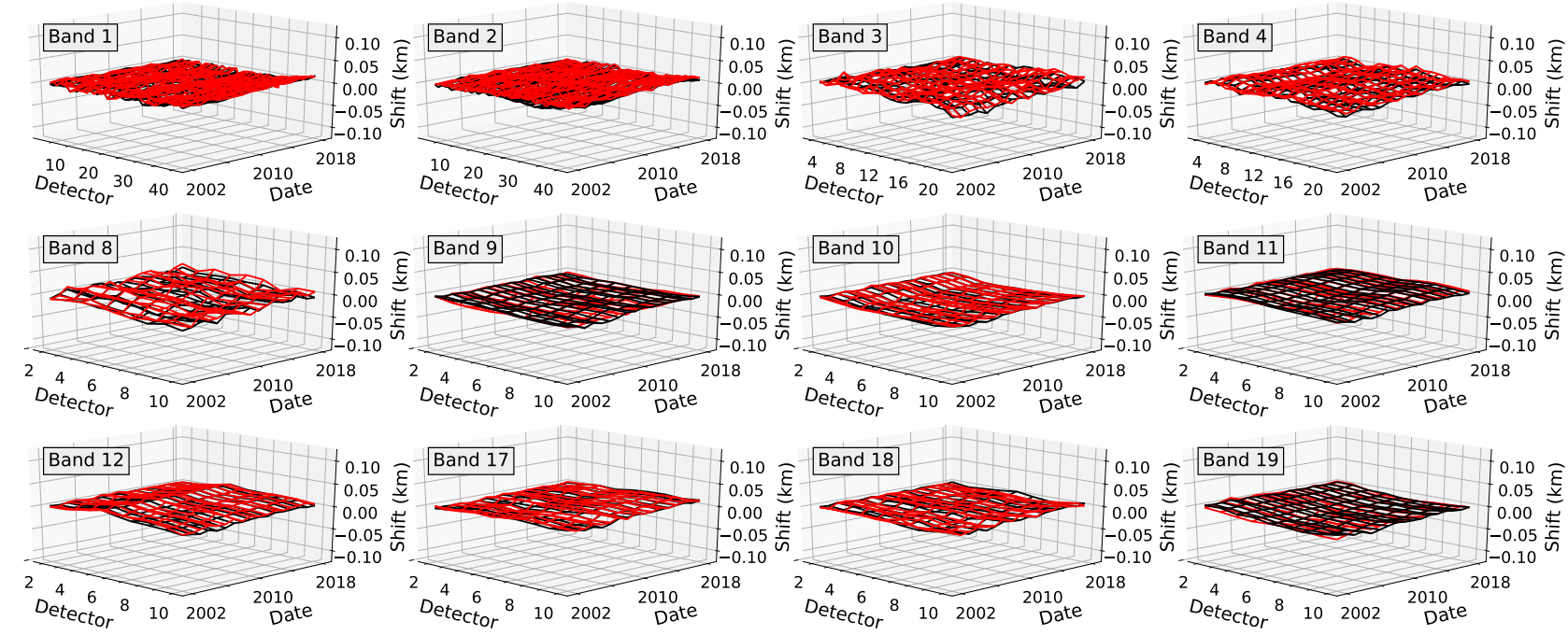

Figure 14. Aqua MODIS DDR along-scan for scheduled (red) and unscheduled (black) lunar observations. The data is the yearly average of all of the observations in each case. Detector 1 is not shown because it is the reference for each of the other detectors.
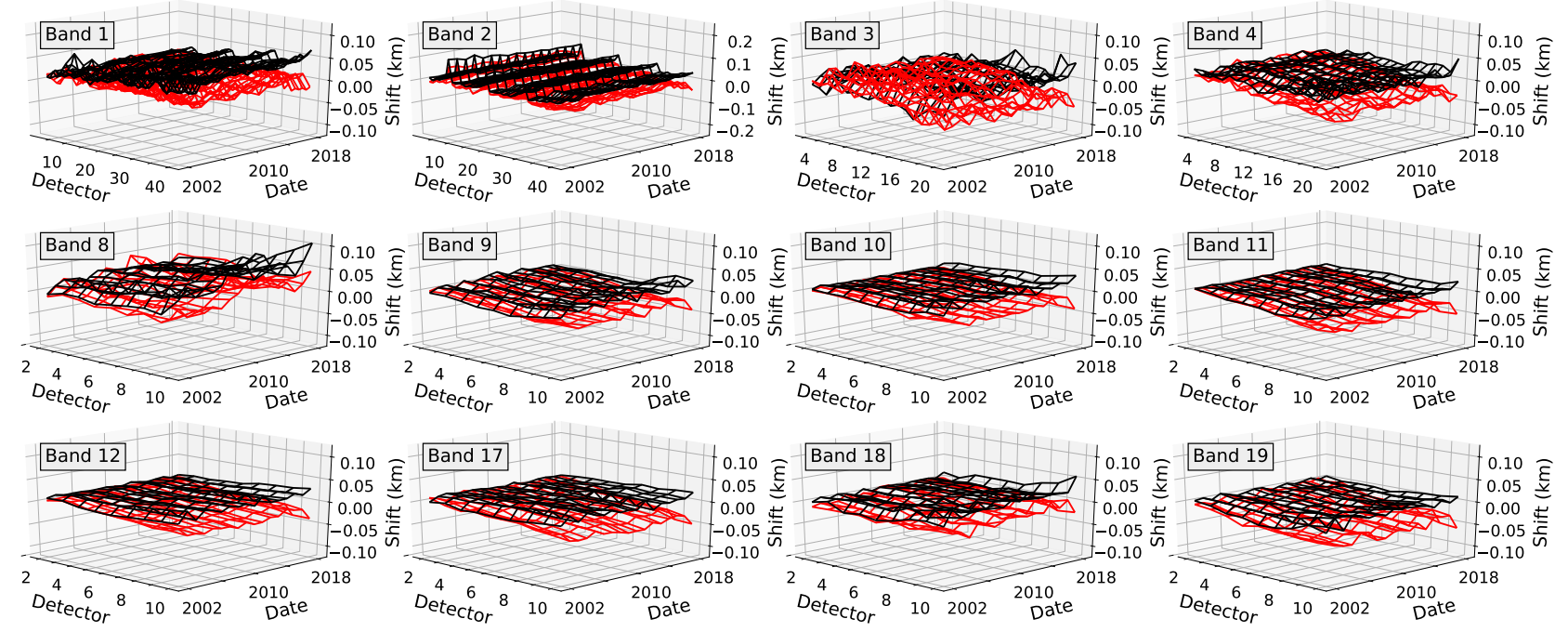

Figure 15. Aqua MODIS DDR along-track for scheduled (red) and unscheduled (black) lunar observations. The data is the yearly average of all of the observations in each case. Detector 1 is not shown because it is the reference for each of the other detectors.

saturated allow us to check the consistency of our saturation correction, which uses the unsaturated signal from band 31 as a reference (similar to the method for bands $13-16$ described previously). During scheduled lunar observations (nominal lunar phase angle between $55-56$ degrees), the lunar signal saturates at approximately, $0.55,0.60,0.65$ and 0.80 of the maximum level for bands $27-30$, respectively. At a lunar phase angle of approximately 80 degrees, the saturation level increases to around 0.8 for bands $27-29$, and band 30 has negligible saturation.

The effect of the correction on the Level-1B data has been analyzed extensively in, ${ }^{9,26}$ and therefore will not be discussed in this work. Also, for use in Collection 6.1, a set of coefficient update criteria is used in order to produce the coefficient time series as opposed to using the coefficient values from each event. Here, we will focus 

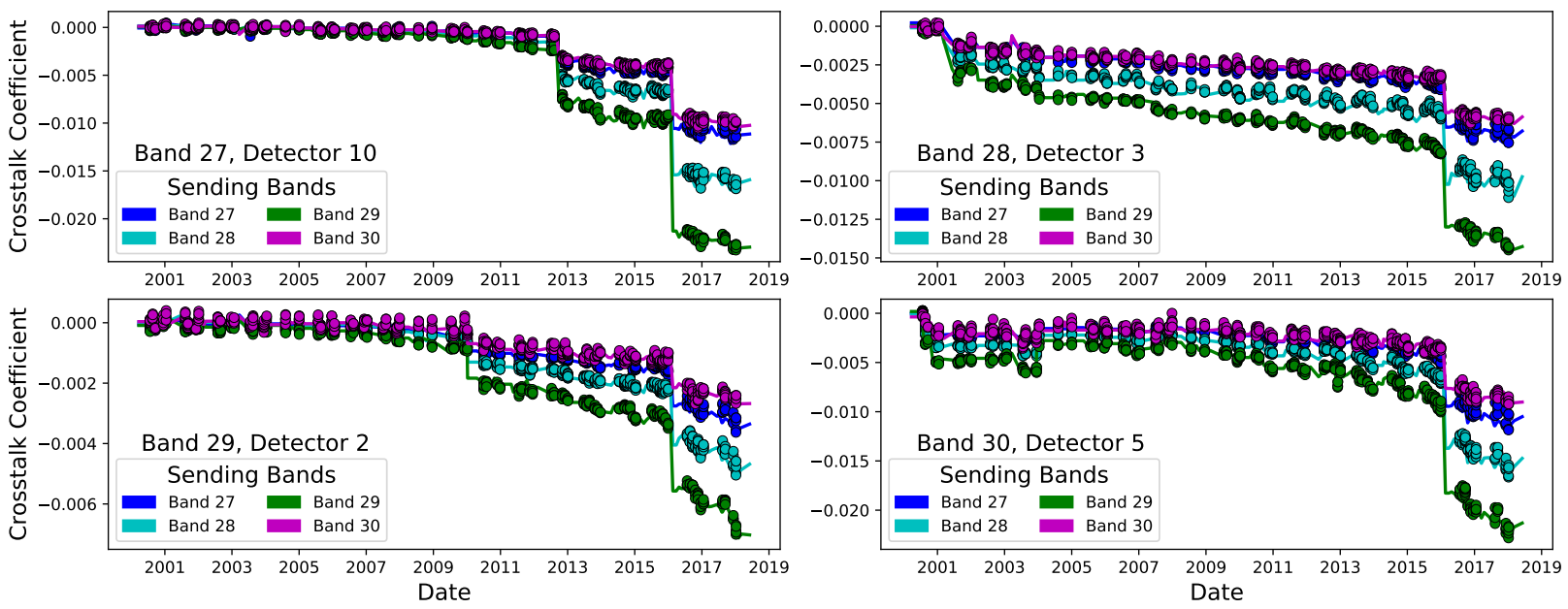

Figure 16. Crosstalk coefficient value comparison for a selected detector in each band, $27-30$. The solid lines show the coefficients from coefficient values from scheduled lunar observations. The circles show the coefficient values from unscheduled lunar observations.

on a comparison of the derived correction coefficient values using data from each scheduled and unscheduled event. In Figure 16, we show the correction coefficients for a selected detector in each band, for all of the corresponding sending bands for both scheduled and unscheduled lunar observations. For each contaminated detector in bands $27-30$, the correction coefficients derived from both sets of observations are in good agreement.

When analyzing the value of the coefficients versus phase angle, similar to the analysis in Figure 6 , we find that for higher phase angle observations, the values of the bands 27 and 28 sending coefficients for some contaminated detectors from unscheduled moons can be a few percent lower in magnitude than the values derived from the scheduled moons for the latest data. This could be the result of the saturation effect discussed previously, as these two bands have a higher saturation for most lunar events than bands 29 and 30. However, this is not expected to have a significant impact on the corrected Earth-view data, as both the signal level for band 29 at typical scene radiance levels and the band 29 coefficient values are higher than those for the other bands. Also, although some large changes in the coefficient values can be seen due to passage through the South Atlantic Anomaly ${ }^{9}$ our analysis indicates that the coefficient values derived from consecutive orbits in unscheduled events are stable from orbit-to-orbit. This analysis shows that unscheduled lunar observations would provide the appropriate assessment of the crosstalk contamination for bands 27 - 30 in the event that a roll maneuver for a lunar observation could not be performed or if additional data is needed in between scheduled observations.

\section{CONCLUSION}

Unscheduled lunar observations provide an opportunity for additional assessments of a variety of on-orbit characterization activities for satellite instruments without the need to schedule any satellite maneuvers. For MODIS, this data can be used to analyze the trending gain of the various spectral bands, spatial characterization, and the impact of electronic crosstalk on the MODIS bands. The gain comparison showed that the scheduled and unscheduled lunar observations were in good agreement, and capture the degradation of the sensor over time. However, this comparison did show some small differences as a function of phase angle at some wavelengths for both Terra and Aqua MODIS, which could potentially lead to a refinement either the modeling or measurement techniques. The spatial characterization results also showed good agreement for scheduled and unscheduled events after the lunar path and instrument attitude corrections were applied. However, the along-track DDR showed some differences between the two that will need to be explored in future work. Finally, a comparison of the electronic crosstalk coefficients derived for bands $27-30$ showed that the unscheduled lunar observations provided consistent coefficient values, which verifies of our previous results over a wider range in phase angle and saturation levels. These observations could be used in Collection 6.1 if data from scheduled lunar observations 
was otherwise unavailable. In the future, we will continue to monitor these sensor performance assessments for unscheduled Moons as the missions continue, and work to develop new ways to exploit these observations.

\section{ACKNOWLEDGMENTS}

The authors would like to acknowledge Daniel Todaro and Michael Lucci of SSAI for their support in obtaining the MODIS attitude data, which was essential for performing the spatial characterization analysis. We would also like to thank Xu Geng of SSAI for his review and comments on this work.

\section{REFERENCES}

[1] Salomonson, V. V., Barnes, W. L., Xiong, X., Kempler, S., and Masuoka, E., "An overview of Earth Observing System MODIS instrument and associated data systems performance," Proc. IGARSS 2, 1174$1176(2002)$.

[2] Xiong, X., Sun, J., Barnes, W. L., Salomonson, V. V., Esposito, J., Erives, H., and Guenther, B., "Multiyear on-orbit calibration and performance of Terra MODIS reflective solar bands," IEEE Trans. Geosci. Remote Sens. 45(4), 879 (2007).

[3] Xiong, X., Wu, A., Wenny, B. N., Madhavan, S., Wang, Z., Li, Y., Chen, N., Barnes, W. L., and Salomonson, V. V., "Terra and Aqua MODIS thermal emissive bands on-orbit calibration and performance," IEEE Trans. Geosci. Remote Sens. 53(10), 5709 (2015).

[4] Xiong, X., Che, N., and Barnes, W. L., "Terra MODIS on-orbit spatial characterization and performance," IEEE Trans. Geosci. Rem. Sens. 43(2), 355-365 (2005).

[5] Xiong, X., Che, N., and Barnes, W. L., "Terra MODIS on-orbit spectral characterization and performance," IEEE Trans. Geosci. Rem. Sens. 44(8), 2198-2206 (2006).

[6] Xiong, X., Sun, J., Xiong, S., and Barnes, W. L., "Using the Moon for MODIS on-orbit spatial characterization," Proc. SPIE 5234, 480 (2004).

[7] Xiong, X., Sun, J., Angal, A., Xie, Y., Choi, T., and Wang, Z., "Results of MODIS band-to-band registration characterization using on-orbit lunar observations," Proc. SPIE 8153, 81531R-1 (2011).

[8] Sun, J., Xiong, X., Madhavan, S., and Wenny, B. N., "Terra MODIS band 27 electronic crosstalk effect and its removal," IEEE Trans. Geosci. Remote Sens. 8, 5722 (2014).

[9] Wilson, T., Wu, A., Shrestha, A., Geng, X., Wang, Z., Moeller, C., Frey, R., and Xiong, X., "Development and implementation of an electronic crosstalk correction for bands 27-30 in Terra MODIS collection 6," Remote Sens. 9(6), 569 (2017).

[10] Sun, J. and Wang, M., "Electronic crosstalk in Aqua MODIS long-wave infrared photovoltaic bands," Remote Sens. 8(10), 806 (2016).

[11] Keller, G. R., Wang, Z., Wu, A., and Xiong, X., "Aqua MODIS band 24 crosstalk striping," IEEE Geosci. Remote Sens. Lett. 14(4), 475 (2017).

[12] Chen, H., Xiong, X., Angal, A., Geng, X., and Wu, A., "Alternative method of on-orbit response-versusscan-angle characterization for MODIS reflective solar bands," J. Appl. Remote Sens. 10(2), 024004 (2016).

[13] Sun, J., Xiong, X., Angal, A., Chen, H., Wu, A., and Geng, X., "Time-dependent response versus scan angle for MODIS reflective solar bands," IEEE Trans. Geosci. Remote Sens. 52(6), 3159-3174 (2014).

[14] Sun, J., Xiong, X., Barnes, W., and Guenther, B., "MODIS reflective solar bands on-orbit lunar calibration," IEEE Trans. Geosci. Remote Sens. 45(7), 2383 (2007).

[15] Sun, J., Xiong, X., and Barnes, W. L., "MODIS reflective solar bands unscheduled lunar observations," Proc. SPIE 6677, 66771K (2007).

[16] Wilson, T. and Xiong, X., "Scheduling observations of celestial objects for Earth observing sensor calibration," Proc. SPIE 10000, 1000011 (2016).

[17] Kieffer, H. H. and Stone, T. C., "The spectral irradiance of the Moon," Astron. J. 129(6), 2887-2901 (1999).

[18] Xiong, X., Sun, J., Fulbright, J., Wang, Z., and Butler, J. J., "Lunar calibration and performance for S-NPP VIIRS reflective solar bands," IEEE Trans. Geosci. Rem. Sens. 54(2), 1052-1061 (2015).

[19] Xiong, X., Geng, X., Angal, A., Sun, J., and Barnes, W., "Using the moon to track MODIS reflective solar bands calibration stability," Proc. SPIE 8176, 817611 (2011). 
[20] Wilson, T., Angal, A., Shrestha, A., and Xiong, X., "Lunar calibration improvements for the short-wave infrared bands in Aqua and Terra MODIS," Proc. SPIE 10423, 1042313 (2017).

[21] Wang, Z., Xiong, X., and Li, Y., "Update of VIIRS on-orbit spatial parameters characterized with the moon," IEEE Trans. Geosci. Rem. Sens. 53(10), 5486-5494 (2015).

[22] Folkner, W., Williams, J., and Boggs, D., "The planetary and lunar ephemeris DE 421," IPN Progress Reports , 42-178 (2009).

[23] Acton, C. H., "Ancillary data services of NASA's navigation and ancillary information facility," Planetary and Space Sci. 44(65) (1996).

[24] Xiong, X., Che, N., Barnes, W., Xie, Y., Wang, L., and Qu, J., "Status of Aqua MODIS spatial characterization and performance," Proc. SPIE 6361, 63610T (2006).

[25] Sun, J., Xiong, X., Che, N., and Angal, A., "Terra MODIS band 2 electronic crosstalk: Cause, impact, and mitigation," Proc. SPIE 7826, 78261Y (2010).

[26] Moeller, C., Frey, R., Borbas, E., Menzel, W. P., Wilson, T., Wu, A., and Geng, X., "Improvements to Terra MODIS L1B, L2, and L3 science products through using crosstalk corrected L1B radiances," Proc. SPIE 10402, 104020O-1 (2017). 